\title{
Synthesis of spirooxindoles with \\ three component reaction between $\beta$-ketoesters, malononitrile and isatin derivatives in the presence of Alum.SiO2 nanoparticles as a new nano catalyst
}

\author{
Marziyeh Rohi, Bahareh Sadeghi *, Mohamadhosaine Moslemin
}

Department of Chemistry, Yazd Branch, Islamic Azad University, P.O. Box 89195-155, Yazd, Iran

\begin{abstract}
In this reseaech, some of the spirooxindole derivaties were prepared via $\beta$-ketoesters, malonoitrile and isatin derivatives in the presence Alum.SiO2 NPs as an effective, recyclable and inexpensive catalyst. The products were identified by IR, 1HNMR, 13C NMR spectra, and melting point. The size of nanoparticles characterized by SEM and TEM techniques. A novel multicomponent synthesis of a series of spirooxindol derivatives is described. The procedures was carried out applying Alum/SiO2 supported on NPS as the novel catalyst giving rise to high to excellent yielded synthetic route of spirooxindol compounds. The major advantages of this protocol are short reaction time ,good yields, simple procedure work up as well as friendly synthesis. In this work, some of the spirooxindole derivaties were prepared via $\beta$ ketoesters, malononitrile and isatin derivatives in the presence Alum.SiO2 NPs as an effective, recyclable and inexpensive catalyst. The products were identified by IR,1HNMR,13CNMR spectra, and melting point. The size of nanoparticles characterized by SEM and TEM techniques.(scheme 1)
\end{abstract}

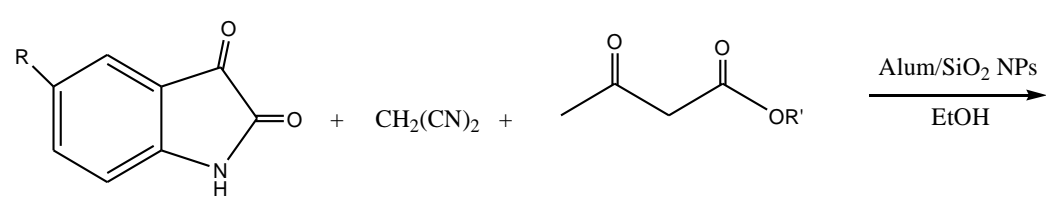<smiles>[R]c1ccc2c(c1)C1(C(=O)N2)C(C#N)=C(N)OC(C)=C1C(=O)O</smiles>

4a: $\mathrm{R}=\mathrm{H}, \mathrm{R}^{\prime}=\mathrm{CH}_{3}$ $4 \mathrm{~b}: \mathrm{R}=\mathrm{NO}_{2}, \mathrm{R}^{\prime}=\mathrm{CH}_{3}$ 4c: $\mathrm{R}=\mathrm{NO}_{2}, \mathrm{R}^{\prime}=\mathrm{C}_{2} \mathrm{H}_{5}$ (scheme 1)

Keywords: Spirooxindoles,Molononitrile,System.Alum.SiO2, nanoparticles . 


\section{Introduction}

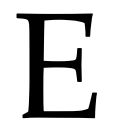

Recently, many research studies have been devoted to the multicomponent reactions (MCR). MCRs are of significant importance from the view point of simplicity and its efficiency since the reactions are widely applied in pharmaceutical chemistry for producing different structures and combinatorial libraries for drug discovery [1]. One of the most applicable structures which have become a privileged skeleton with broad and promising activities in various therapeutic areas is spirooxindole compounds [1].Nowadays, enantioselective synthesis of spirooxindoles via efficient catalysts has been developing utilizing novel catalyst systems[2-16].Bergman at coworkers synthesized various derivatives of mono spirooxindol via one pot reaction of isatin and some alpha-amino acids in methanol and water[17].Also, Yau et al. reported the MCR reaction of isatin, pyrolidin, 2- carboxilic acid and molonat esters to furnish spirooxindols in good yieds [18].In addition, Yang and coworkers utilized four-component condensation between aromatic aldehyd , 1,3 indandion, sarcozin and isatin to prepare a series of di spiropirolidin compounds via knovenagol reaction [19].

Experimental:The chemicals used in this work were purchased from sigma-Aldrich and Fluka and were used without furher purification. stable silica gel nanoparticles were readily prepared as described elsewhere [8] and used for preparation of the catalyst(Alum/SiO2NPS).IR spectra were recorded on a shimadzu IR-470 spectrometer as $\mathrm{KBr}$ discs. The NMR spectra were obtained on a broker Avance DRX-400 FT spectrometer (1H NMR at 400HZ, 13CNMR at $100 \mathrm{HZ}$ ) with DMSO-D6 as solvent and TMS as internal standard. Melting point were determined with an Electro thermal 9100 apparatus. Elemental analysis was performed in the analytical laboratory of the Science and Research unit of Islamic Azad University. The morphology of the catalyst was observed by scanning electron microscopy (SEM) with a VEGA/TESCAN, using an accelerating Voltage of $15 \mathrm{kv}$.

In this research, derivatives $(4 a),(4 b),(4 c)$ using reaction triple-component between beta keto acids, malononitril and isatin derivatives in the presence of nano-catalyst was produced. This is an effective, recoverable and cheap way. The spirooxindol derivatives are included in numerous and they are dominant molecules in medicinal chemistry, sush as ptropodine, horsfiline, isopteropodine and spirotryprostatin[2]. On the basis of biological studies, the existence of two different heterocyclic moieties in a single molecule often show enhances the biological activity dramatically[3].In this article, we report a simple and efficient method for synthesis of spirooxindoles, through a three component reaction using isatins, $\beta$-ketoesters and malonitrile in the presence of Alum/SiO2 NPs under reflux in ethanol.(Scheme2-3)

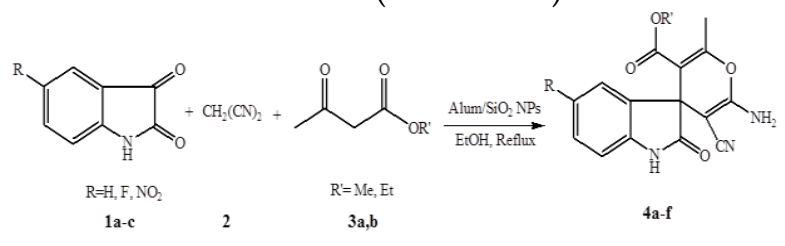

(Scheme2)

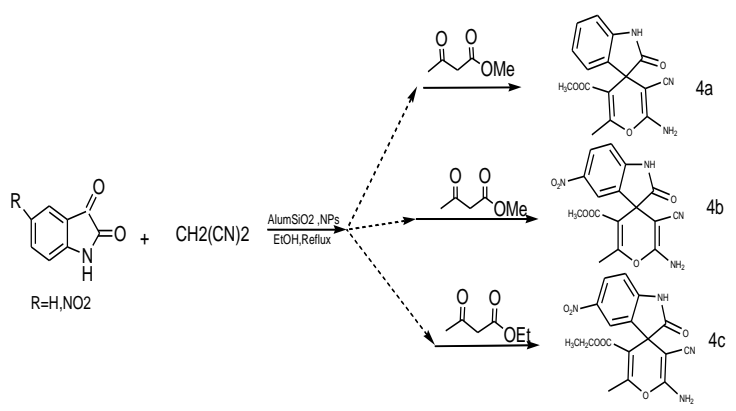

(Scheme 3) 


\begin{tabular}{|c|c|c|c|c|c|}
\hline $\begin{array}{l}\text { MP: } \\
(\dot{\mathrm{c}})\end{array}$ & $\begin{array}{l}(\%) \\
\text { Yeil } \\
\text { d) }\end{array}$ & $\begin{array}{l}\text { Produ } \\
\text { cts }\end{array}$ & $\begin{array}{l}\beta \\
\text { keto } \\
\text { Este } \\
r\end{array}$ & $\begin{array}{l}\text { Isatin } \\
\text { drivativ } \\
\text { es }\end{array}$ & entry \\
\hline 298 & $5^{8}$ & $4 a$ & $3 a$ & & 1 \\
\hline 295 & $8^{8}$ & $4 b$ & $3 a$ & & 2 \\
\hline 293 & 90 & & $3 b$ & & \\
\hline
\end{tabular}

(Table.1)

synthesis of spirooxindole derivatives in the presence of alum.SiO2 NPS Catalyst:

To a suspension of $0.75 \mathrm{~g}$ of silicagel nanoparticles in $10 \mathrm{ml}$ of water $0.25 \mathrm{~g}$ $\mathrm{KAl}(\mathrm{SO} 4) 2.12 \mathrm{H} 2 \mathrm{O}$ was added. The suspension stirred at room temperature for $6 \mathrm{~h}$, then water was evaporatorated under reduced pressure for $20 \mathrm{~min}$ and the residue dried at $60 \mathrm{oC}$ for 3h.Alum $/ \mathrm{SiO} 2$ nanoparticles $(0.08 \mathrm{~g})$ was added to a stirred mixture of the isatin derivatives $(1 \mathrm{mmol}), \beta$-ketoesters $(1 \mathrm{mmol})$ and malonitrile $(1$ $\mathrm{mmol})$ in $\mathrm{EtOH}(5 \mathrm{~mL})$. the materials were mixed and refluxed for the $15 \mathrm{~min}$. The progress of the reaction was followed by TLC (n-hexane:ethyl acetate). After completion of the reaction, the mixture was filtered to remove the catalyst. After evaporation of the solvent, the crude product was re crystallised from hot ethanol to obtain the pure compound. (Table1-2)

\begin{tabular}{|c|c|c|c|c|}
\hline \multicolumn{2}{|r|}{ Catalys } & \multicolumn{2}{|c|}{ Time(min) } & Yield \\
\hline 1 & $\begin{array}{ll}\mathrm{InCl} 3 & (20 \mathrm{~mol} \%)\end{array}$ & $\begin{array}{l}\mathrm{CH} 3 \mathrm{CN} \\
\text { /reflux }\end{array}$ & 90 & 75 \\
\hline 2 & TEBAa (20 mol\%) & $\mathrm{H} 2 \mathrm{O} / 60^{\circ} \mathrm{C}$ & 120 & 94 \\
\hline 3 & $\begin{array}{c}\text { Sodium stearat (10 } \\
\text { mol\%) }\end{array}$ & $\mathrm{H} 2 \mathrm{O} / 60^{\circ} \mathrm{C}$ & 180 & 95 \\
\hline 4 & I2 (10 mol\%) & $\mathrm{H} 2 \mathrm{O} / 50^{\circ} \mathrm{C}$ & 60 & 80 \\
\hline 5 & $\begin{array}{c}\mathrm{HAuCl} 4.3 \mathrm{H} 2 \mathrm{O}(5 \\
\mathrm{mol} \%)\end{array}$ & $\begin{array}{c}\text { PEGb } 400 \\
/ 70^{\circ} \mathrm{C}\end{array}$ & 30 & 96 \\
\hline 6 & $\mathrm{NH} 4 \mathrm{Cl}(20 \mathrm{~mol} \%)$ & $\mathrm{H} 2 \mathrm{O} / 80^{\circ} \mathrm{C}$ & 10 & 92 \\
\hline 7 & $\begin{array}{c}\mathrm{MgO} \text { nanocrystallin } \\
(15 \mathrm{~mol} \%)\end{array}$ & $\mathrm{H} 2 \mathrm{O} / 80^{\circ} \mathrm{C}$ & 120 & 95 \\
\hline 8 & $\begin{array}{c}\mathrm{KAl}(\mathrm{SO} 4) 2.12 \mathrm{H} 2 \mathrm{O} \\
(0.08 \mathrm{~g})\end{array}$ & EtOH/reflux & 15 & 60 \\
\hline 9 & $\begin{array}{c}\text { Alum/SiO2 } \\
\text { nanoparticles }(0.08 \mathrm{~g})\end{array}$ & EtOH/reflux & 15 & 96 \\
\hline
\end{tabular}

(Table.2)

Characterization of the synthesized compounds:

4b:6-amino-3-methyl-1-phenyl-spiro[( $\left.3^{\prime} \mathrm{H}\right)$ indole-3, $4^{\prime} 4(\mathrm{H})$-pyrano[2,3-d]pyrazole]-( $\left.1^{\prime} \mathrm{H}\right)-2^{\prime}-$ one-5-carbonitrileDark red ,crystal; mp 258260C;IR(KBR):3455,3295,3175,2190,1696,1649,112 3,749

4d:2-Amino-5-oxo-spiro[(4H)-5,6,7,8tetrahydrochromene-4, $3^{\prime}-\left(3^{\prime} \mathrm{H}\right)-5^{\prime}$-Fluoro-indol](1H)-2'-one-3-arbonitrile White, solid; $\mathrm{mp}$ 289291c;IR(KBr):3345,3095,2960,2190,1702,1665,1604, 1483,1348,1211,1013,803,

1HNMR(DMSO-d6,400MHz)ס:10.43

$\mathrm{IH}, \mathrm{NH}), 7.30(\mathrm{sbr}, 2 \mathrm{H}, \mathrm{NH} 2), 6.947 .01(\mathrm{~m}, 2 \mathrm{H}, \mathrm{ArH}), 6$. $78(\mathrm{~d}, \mathrm{IH}, \mathrm{J}=4 \mathrm{~Hz}), 2.63-2.67(\mathrm{~m}, 2 \mathrm{H}, \mathrm{CH} 2), 2.22-$

2.26(m,2H,CH2),1.90-1.95(m,2H,CH2),13C NMR(DMSO

d6,MHz)ঠ:195.10,178.14,166.37,159.30,156.95,138. $15,136.24,117.22,114.407,114.17,111.33,109.77,56.8$ $9,47.33,36.26,26.73,19.68$

4e:

2-Amino-5-0x0-spiro[4,5,6,7,8tetrahydrocyclopenta(b)pyran $\quad-444,3^{\prime}-\left(3^{\prime} \mathrm{H}\right)-5^{\prime}-$ Fluoro-indol]-(1'H)-2'-one-3-carbonitrileBright pink, Solid; mp 313-316C;IR 9KBr):3315, 3060, 2995, 2199, 1712, 1673, 1601, 1484, 1344, 1231 1009, 873, 1H NMR (DMSO-d6, 400MHz) $\delta:$ 10.61(s, $1 \mathrm{H}, \mathrm{NH}), 7.58(\mathrm{sbr}, 2 \mathrm{H}, \mathrm{NH} 2), 7.04-7.07(\mathrm{~m}$, $1 \mathrm{H}, \operatorname{ArH}), 7.01(\mathrm{~d}, 1 \mathrm{H} \mathrm{J}=2.4, \operatorname{ArH}), 6.83(\mathrm{~d}, 1 \mathrm{H}$, $\mathrm{J}=4.4, \mathrm{ArH}), 2.82(\mathrm{dd}, 2 \mathrm{H}, \mathrm{J}=4.8 \mathrm{~Hz}, \mathrm{~J}=4 \mathrm{~Hz}, \mathrm{CH} 2)$, 2.39(t, 2H, J=4.8Hz, CH2) 13C NMR(DMSO-d6, 100MHZ) 8:199.80, 177.81, 160.53, 157.09, 138.01, 
133.66, 117.39, 115.03, 114.30, 112.22, 111.97, $110.14,55.88,46.99,33.13,24.90$

4f: 2-Amino 5,7-dioxo-sprino[(3')-5'-fluoroindol-3',4,4(H)-5,6,7,8-tetrahydropyrano(2,3-

d)pyrimidine]-(1'

H)-2'-one-carbonitrile

White,solid;mp246-250

c';IR(Kbr):3395,3160,2200,1716,1692,,1671,1483,13

90,1223,1108,796, 1H NMR (DMSOd6,400MHz)\&: 12:31 (s, 1H,NH),11.140(S 1H, $\mathrm{NH}), 10.49(\mathrm{~s}, 1 \mathrm{H}, \mathrm{NH}), 7.42(\mathrm{~s}, 2 \mathrm{H}, \mathrm{NH} 2), 7.15(\mathrm{~d} \mathrm{~d}$ , $1 \mathrm{H}, \mathrm{J}=2.6, \mathrm{~J}=8.2), 6.96(\mathrm{dd}, 1 \mathrm{H}, \mathrm{J}=2.68, \mathrm{~J}=1.08 \mathrm{~Hz}$ ), $6.75 \mathrm{~d} \mathrm{~d}, 1 \mathrm{H}, \mathrm{J}=4.36 \mathrm{~Hz}, \mathrm{~J}=4.28 \mathrm{~Hz}), 13 \mathrm{C} \mathrm{NMR}$ (DMSO-d6, 100MHz) \&:178.18 (c=o), 161.93, $159.92(\mathrm{c}=\mathrm{o}), 158.78,157.57,149.70,138.77,117.29$, 115.05,112.19(CN), 110.33(CN), 86.84,56.98

4g: 2-amino-5-benzoyl-spiro[pyrano-4,3-(3H)5-fluoro-indole]-(1H)-2-one-6-phenyl 3carbonitrile Dark pink,Crystal,mp 242-246C;IR (KBr):3990,3380,3155,2185,1722,1675,1649,1485,13 46,1223,1054,805,1 HNMR(DMSO-d6,400 ,MHZ)ठ:10.77(s,1H.NH),7.79(d,2H,J=7064,arom)7 $.62(\mathrm{~s}, 2 \mathrm{H}, \mathrm{NH} 2), 7.51(\mathrm{t}, 2 \mathrm{H}, \mathrm{j}=6.04$, arom $), 7036(\mathrm{t}, 1 \mathrm{H}$, $\mathrm{j}=4.96 \mathrm{HZ}$, arom $), 7.20(\mathrm{dd}, 1 \mathrm{H}, \mathrm{j}=2.64, \mathrm{j}=6.04, \mathrm{j}=8.08 \mathrm{HZ}$ ,arom $) 7.12 \quad(\mathrm{dt}, \quad, 1 \mathrm{H}, \mathrm{J}=16.0, \mathrm{~J}=2.6$ HZ, arom), 6.94(dd,1H, $\quad \mathrm{j}=8.52 \mathrm{HZ}, \mathrm{j}=4.28$ $\mathrm{HZ}$, arom),1.59(s,3H,CH3)13 C NMR (DMSO$\mathrm{d} 6,100$

NMR) ठ:178,161.51,160.30,157.93,145.46,144.30,138.19,13 7.69,134.48,129.87,127.07,118.33,116.09,113.31,113. $07,111.26,96.30,56.15,12.18$

4h: Phenyl2'-Amino-3'-cyano-6'-phenol-2-oxo2-hydrospiro[5-fluoro-indol-3,4'-Pyran]-5'methanone

Dark pink,Crystal,mp 242246C;IR(KBr):3280,2225,1730,1589,1482,1346,1178 ,1100,1H NMR ((DMSO-d6,400 M HZ) $\delta: 11.24(\mathrm{~S}, 2 \mathrm{H}, \mathrm{NH} 2), 8018(\mathrm{M}, 2 \mathrm{H}$, arom $), 817(\mathrm{~d}$, $1 \mathrm{H}, \mathrm{J}=1.44 \mathrm{HZ}$, arom),7.55(td,3H, J=4.3 HZ, , $\mathrm{J}=2.64 \mathrm{HZ}$, arom), $7.45(\mathrm{td}, 3 \mathrm{H}, \mathrm{J}=9.2 \mathrm{HZ}$, , J=2.64 $\mathrm{HZ}$ ， arom),7.35(S-br,1H,NH),6.97(d,2H, J=4.32 $\mathrm{HZ}$, arom),6.95(d,2H, J=4.28 HZ, arom),13C NMR (DMSO-d6,100 M HZ) ঠ:164.15(C=O),159.15(C=O),156.77,150.68,150.64,1 43.47,143.45,133.50 ,129.32,127.88,125.02, 124.79, $119.70,119.61, \quad 113.38, \quad 113.33, \quad 112.53$, 112.27,111.67(CN), 93.71,82.73

4i: 2Amino-5oxo-spiro[(4H)-indole(1,2-b)pyran4,3'-(3'H)-5'-Fluoro-indol]-(1'H)-2' -one-3carbonitrile
Dark yellow,Solid;mp 286-288c; Ir (kBr): 3305, 3190, 2955, 2195, 1729, 1666, 1603,1485, 1334, 1179, 1037, 924; 1H NMR (DMSO-d6, 400MHz) $8: 10.73(\mathrm{~s}, 1 \mathrm{H}, \mathrm{NH}), 7.78(\mathrm{sbr}, \quad 2 \mathrm{H}$, $\mathrm{NH} 2), 7.58(\mathrm{td}, \quad 1 \mathrm{H}, \quad \mathrm{J}=6.8 \mathrm{~Hz}, \quad \mathrm{~J}=1.2 \mathrm{~Hz})$, $7.38(\mathrm{~d}, 1 \mathrm{H}, \mathrm{J}=6.8 \mathrm{~Hz}), 7.32(\mathrm{~d}, 1 \mathrm{H}, \mathrm{J}=7.2 \mathrm{~Hz}), 7.28(\mathrm{dd}$, $1 \mathrm{H}, \mathrm{J}=8 \mathrm{~Hz}, \mathrm{~J}=2.8 \mathrm{~Hz}), 7.08(\mathrm{td}, 1 \mathrm{H}, \mathrm{J}=8.8 \mathrm{~Hz}, \mathrm{~J}=2.8$ $\mathrm{Hz}), 6.90(\mathrm{~d}, 1 \mathrm{H}, \mathrm{J}=4.4 \mathrm{~Hz}) 13 \mathrm{C}$ NMR(DMSO-d6, $100 \mathrm{MHz}) \mathrm{\delta}: 1899.32,160.46,159.57,157.22,137.96$, $136.29,135.10,133.63,131.35,130.48,122.75$, $118.84,117.22,117.22,115.61,115.38,112.69$, $112.45,110.35,110.48$

\section{Results and Discussion:}

In summary, Alum/SiO2 nanoparticles is synthesized and have shown that it has advantages in the preparation of spirooxindole derivatives such as shorter reaction times, simple work-up, and affords excellent yield. The present method does not involve any hazardous organic solvent. Therefore, this procedure could be classified as green chemistry. In continuation of our previous research on the use of solid acids in organic synthesis, 4,5 the synthesis of Alum/SiO2 NPs as a new catalyst have been investigated and applied for the synthesis of spirooxindole derivatives, by the condensation of an isatins, $\beta$ ketoesters and malonitrile.

Mechanism:

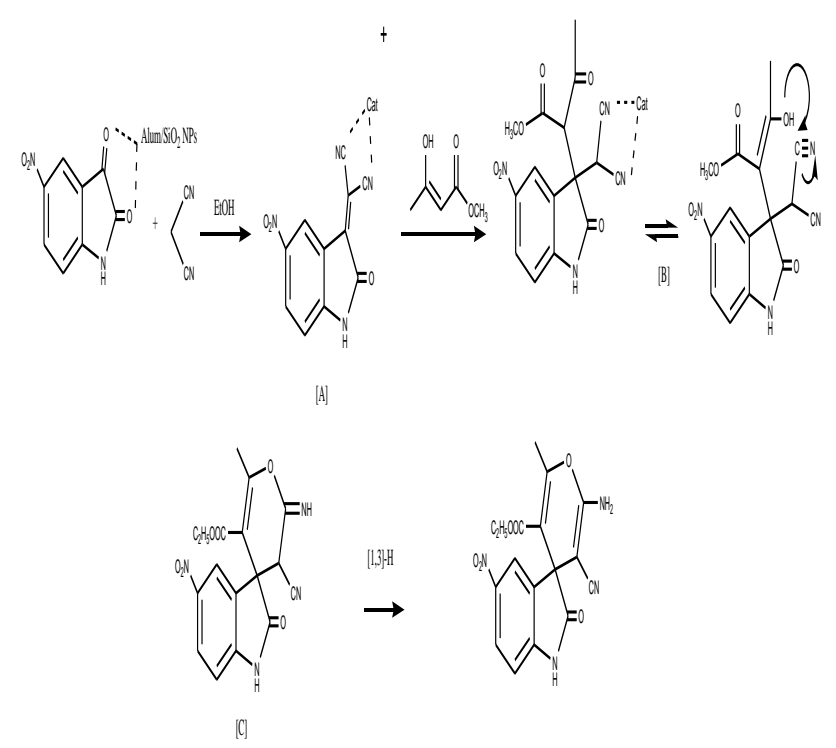

\section{Conclusion:}

The stable catalyst is easily prepared and used for preparation of spirooxindole derivatives. The dimensions of nanoparticles were observed with SEM. As shown in Figure 1, the size of commercial Alum/SiO2 nanoparticles 
are about 23-30 $\mathrm{nm}$. To optimize the reaction conditions, the reaction of isatin, ethylacetoacetate and malonitrile was used as a model reaction. In order to establish the better catalytic activity of Alum/SiO2 nanoparticles, the model reaction has compared with other catalysts reported in literature. To study the scope of the reaction, a series of $\beta$-ketoesters and isatin derivatives with malonitrile were examined by Alum/SiO2 nanoparticles as catalyst. An interesting feature of this method is that the reagent can be regenerated at the end of the reaction and can be used several times without losing its activity. Alum $/ \mathrm{SiO} 2$ nanoparticles were prepared as a new catalyst and shown to have advantages in the preparation of spirooxindole derivatives,including shorter reaction time,high yields, and simple work-up.This method does not involve use of hazardous organic solvent, so it can beclassified as green chemistry.

Figure 1. SEM images of Alum/SiO2 nanoparticles
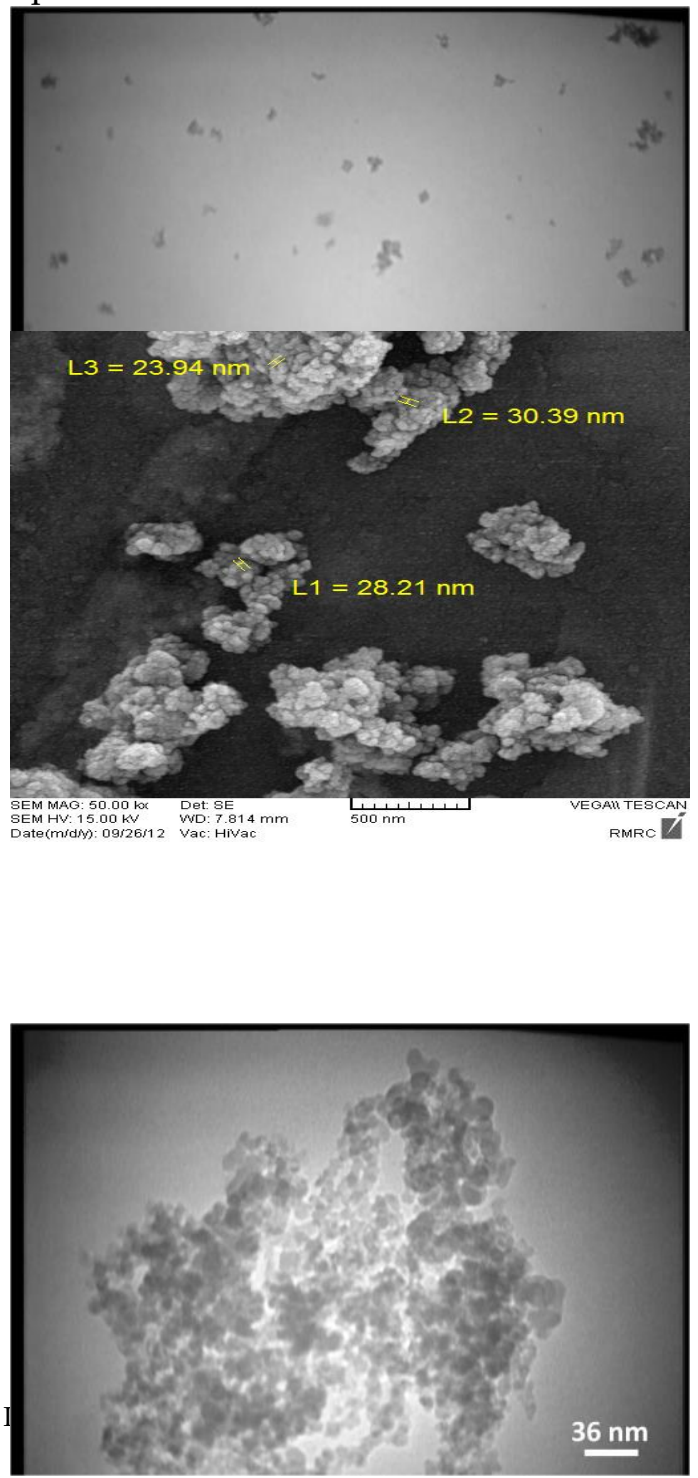

Figure 2. spectroscopy images of (spirooxindole derivatives)

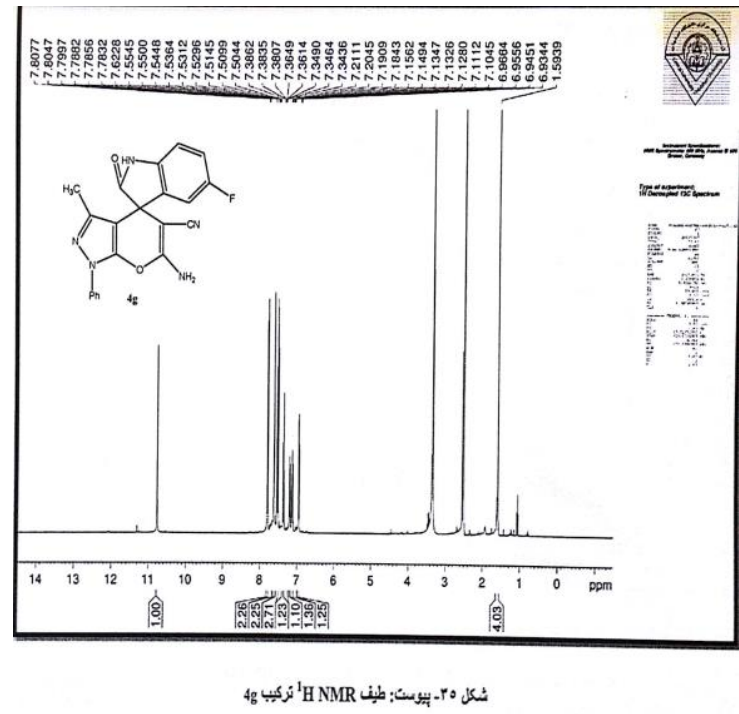

\section{ACKNOWLEDGEMENTS}

The research Council of the Islamic Azad University of Yazd is gratefully acknowledged for the financial support for this work

\section{Refrences:}

1. Pandeya, S. N.; Sriram, D.; Nath, G.; DeClercq, E. Farmaco, 1999, 54, 624.and Armstrong, R.W.; Combs, A.P.; Tempest, P.A.; Brown S.D.; Keating, T.A. Acc. Chem. Res., 1996, 29, 123.

2. Daisley, R. W.; Shah, V. K. J. Pharm. Sci., 1984, 73, 407. And Kang, T.-H.; Matsumoto, K.; Murakami, Y.; Takayama, H.; Kitajima, M.; Aimi N.; Watanabe, H. Eur. J. Pharmacol., 2002, 444, 39.

3. Piscopo, B.; Diumo, M. V.; Godliardi, R.; Cucciniello, M.; Veneruso, G. Bol. Soc. Ital. Biol. Sper., 1987, 63, 827.and Ballini, R.; Bosica, G.; Conforti, M. L.; Maggi, R.; Mazzacanni, A.; Righi P.; Sartori, G. Tetrahedron, 2001, 57, 1395.

4. Sadeghi, B. J. Chem. Res. 2013, 37, 171. And Pandeya, S. N.; Sriram, D.; DeClercq, E.; Pannecouque, C.; 
Witvrouw, M. Indian J. Pharm. Sci., 1998, 60, 207. General Papers ARKIVOC 2007 (xiv) 51-57 ISSN 1424-6376 Page 57 (CARKAT USA, Inc.

5. . Sadeghi, B.; Mirjalili, B. F.; Bidaki S, and Ghasemkhani, M. J. Iran. Chem. Soc. 2011, 8, 648.and Pandeya, S. N.; Sriram, D.; DeClercq, E.; Nath, G. Eur. J. Pharm. Sci., 1999, 9, 25.

6. Pandeya, S. N.; Sriram, D.; DeClercq, E.; Nath, G. Pharm. Acta. Helv., 1999, 74, 11.

7. Pandeya, S. N.; Yogeswari, P.; Sriram, D.; DeClercq, E.; Pannecouque, C.; Witvrouw, M. Chemotherapy, 1999, 45, 192.

8. Pandeya, S. N.; Sriram, D.; DeClercq, E.; Nath, G. Arzneim Forsch., 2000, 50, 55.

9. Selvam, P.; Chandramohan, M.; DeClercq, E.; Witvrouw, M.; Pannecouque, C. Eur. J.

10. Pharm. Sci., 2001, 14, 313.

11. Gursoy, A.; Karali, N.; Buyuktimakin, S.; Demirayak, S.; Ekinci, A. C.; Ozer, $\mathrm{H}$.

a. Batanero, B.; Barba, F. Tetrahedron Lett. 2006, 47, 8201. (b) Deng, H.; Konopelski, J. P.Org. Lett. 2001, 3, 3001. (c) Jahng, K. C.; Kim, S. I.; Kim, D. H.; Seo, C. S.; Son, J. -K.; Lee,

12. S. H.; Lee, E. S.; Jahng, Y. Chem. Pharm. Bull. 2008, 56, 607. (d) Kitajima, M.; Mori, I.; Arai, K.; Kogure, N.; Takayama, H. Tetrahedron Lett. 2006, 47, 3199. (e) Lee, E. S.; Park, J. -G.; Jahng, Y. Tetrahedron Lett. 2003, 44, 1883. (f) Overman, L. E.; Peterson, E. A. Angew. Chem. Int. Ed. 2003, 42, 2525. (g) Sun, C.; Lin, X.; Weinreb, S. M. J. Org. Chem. 2006, 71, 3159. (h) Torres, J. C.; Pinto, A. C.; Garden, S. J. Tetrahedron 2004, 60, 9889. (i) Trost, B.; Brennan, M. Synthesis 2009, 3003. 15. (a) Aboul-Fadl, T.; BinJubair, F. A. S.; Aboul-Wafa, O. Eur. J. Med. Chem. 2010, 45, 4578. (b) Gupta, L.; Sunduru, N.; Verma, A.; Srivastava, S.; Gupta, S.; Goyal, N.; Chauhan, P. M. S. Eur. J. Med. Chem. 2010, 45, 2359. (c) Shibinskaya, M. O.; Lyakhov, S. A.; Mazepa, A. V.; Andronati, S. A.; Turov, A. V.; Zholobak, N. M.; Spivak, N. Y.
Eur. J. Med. Chem. 2010, 45, 1237. (d) Bandekar, P. P.; Roopnarine, K. A.; Parekh, V. J.; Mitchell, T. R.; Novak, M. J.; Sinden, R. R. J. Med. Chem. 2010, 53, 3558. (e) Bhattacharjee, A. K.; Skanchy, D. J.; Reviews and Accounts ARKIVOC 2012 (i) 277-320 Page 316 CARKATUSA, Inc. Jennings, B.; Hudson, T. H.; Brendle, J. J.; Werbovetz, K. A. Bioorg. Med. Chem. 2002, 10, 1979. (f) Nguyen, Q. -D.; Aboagye, E. O. Integr. Biol. 2010, $2,483$.

a. Doménech, A.; DoménechCarbó, M. T.; Sánchez del Río, M.; Vázquez de Agredos Pascual, M. L.; Lima, E. New J. Chem. 2009, 33, 237. (b) Ferreira, E. S. B.; Hulme, A. N.; McNab, H.; Quye, A. Chem. Soc. Rev. 2004, 33, 329.

13. Rehn, S.; Bergman, J.; Stensland, B. Eur. J. Org. Chem. 2004, 2004, 413.

14. Xie, Y. M.; Yao, Y. Q.; Sun, H. B.; Yan, T. T.; Liu, J.; Kang, T. R. Molecules 2011, 16, 8745.

15. Li, M.; Yang, W. -L.; Wen, L. -R.; Li, F. -Q. Eur. J. Org. Chem. 2008, 2008, 2751.

16. Xie, Y. M.; Yao, Y. Q.; Sun, H. B.; Yan, T. T.; Liu, J.; Kang, T. R. Molecules 2011, 16, 8745.

17. Lee, k.; Sathyagal A.N.;.Mc Cormick, A.V. Colloids surface A, 1998, $144,115$.

18. Li, M.; Yang, W. -L.; Wen, L. -R.; Li, F. -Q. Eur. J. Org. Chem. 2008, 2008, 2751.

19. Lee, k.; Sathyagal A.N.;.Mc Cormick, A.V. Colloids surface A, 1998, 144, 115. 